Artículo

\title{
Diversificación de ingresos de la agricultura familiar durante 2018 en Tehuatzingo, Libres, Puebla
}

\author{
Margarita Arteaga Domínguez ${ }^{1}$ \\ Primo Sánchez Morales ${ }^{1 \S}$ \\ Omar Romero Arenas ${ }^{1}$ \\ Ignacio Ocampo Fletes ${ }^{2}$ \\ Antonio Rivera Tapia ${ }^{3}$ \\ Israel Gerardo García Pérez ${ }^{4}$
}

${ }^{1}$ Maestría en Manejo Sostenible de Agroecosistemas-Centro de Agroecología del Instituto de CienciasBenemérita Universidad Autónoma de Puebla. Carretera San Baltazar Tetela, Edificio Val 1 km 1.7, San Pedro Zacachimalpa, Puebla. CP. 71960. (arteaga.mag@gmail.com; biol.ora@ hotmail.com). ${ }^{2}$ Colegio de Postgraduados-Campus Puebla. Boulevard Forjadores de Puebla núm. 205, Santiago Momoxpan, San Pedro Cholula, Puebla. CP. 72760. (agroecología_iof@yahoo.com). ${ }^{3}$ Centro de Investigaciones en Ciencias Microbiológicas-Instituto de Ciencias-Benemérita Universidad Autónoma de Puebla. Ciudad Universitaria, Edificio IC11. CP. 72570. (jart70@yahoo.com). ${ }^{4}$ Facultad de Economía-Benemérita Universidad Autónoma de Puebla. Calle la academia, entre Av. Universidad y Av. San Claudio. CP. 72592. (gerardo.garciaperez@correo.buap.mx).

§Autor para correspondencia: primosamo@yahoo.com.

\section{Resumen}

La pluriactividad es un fenómeno actual en los hogares rurales. El objetivo de este trabajo fue analizar las fuentes de ingreso de familias campesinas en un contexto de agricultura familiar (AF) de subsistencia debido a la importancia de satisfacer primero las necesidades alimenticias en el hogar. La información primaria se obtuvo vía encuesta $(\mathrm{N}=90$ y $\mathrm{n}=46)$ a través de un muestreo aleatorio simple con productores agrícolas de maíz y calabaza en el ejido Tehuatzingo, Libres, Puebla. Se estudiaron dos sistemas de manejo: el sistema en monocultivo (SMo) y el sistema milpa modificado (SMM). Más de 50\% de la fuerza de trabajo en los dos grupos (58\% en el SMM y $61 \%$ para el SMo) se encuentra relacionada con el trabajo familiar. La actividad de los productores es considerada pluriactiva (más de la mitad de sus ingresos derivan de actividades no-agrícolas), tal es el caso de los productores del SMo. Sucede lo contrario con SMM donde se estima que $46 \%$ de sus ingresos derivan de actividades fuera de la parcela agrícola; por lo tanto, es considerada como AF especializada (más de la mitad de sus ingresos provienen de actividades agrícolas). Se concluye que el grupo con mayor tendencia a la diversificación de fuentes de ingreso es SMM, tal como lo refleja el Î́ndice de Simpson donde el ingreso agrícola es mayor por la diversificación y venta de cultivos. Esto contrasta con el SMo que obtiene mayor remuneración económica fuera de la parcela agrícola.

Palabras clave: desagrarización, ingresos diversificados, pluriactividad.

Recibido: febrero de 2021

Aceptado: abril de 2021 


\section{Introducción}

Con la apertura comercial y las diferentes reformas, tales como la privatización de tierras en México, a partir de 1994 se previó que la agricultura familiar (AF) desaparecería, pues se tuvo la idea de que la producción pasaría a ser competitiva y se ignoró el potencial de los agricultores minifundistas para producir alimentos (Yúnez et al., 2013). A pesar de la persistencia de la pobreza rural y el incremento en la demanda de alimentos en Latinoamérica (Schneider, 2014) debe reconocerse que este tipo de agricultura es la principal proveedora de la mayoría de los alimentos básicos del consumo humano (Schejtman, 2008; García et al., 2016).

Es primordial para la seguridad alimentaria en el planeta, y en su mayoría apegada a los principios de producción agroecológica (Alves y Días, 2019; Bosc y Sourisseau, 2019). Además, este modo de agricultura reivindica al campesinado debido a que resalta las prácticas que se han visto obligados a realizar para garantizar su reproducción social y económica (Schneider, 2003; Woods, 2014).

La AF, a diferencia de la agricultura capitalista, se caracteriza principalmente por la índole familiar en la organización del trabajo y el acceso escaso a la tierra (Sabourin et al., 2015; Orsini et al., 2018). Existe una clasificación de la AF según la Food and Agriculture Organization (FAO) y el Banco Interamericano de Desarrollo (BID), en la que se consideran tres tipos: agricultura familiar de subsistencia (AFS) donde predomina el autoconsumo con una tendencia hacia el empleo asalariado como búsqueda de medios de sobrevivencia. Agricultura familiar en transición (AFT) donde la producción se destina a la venta y al autoconsumo, contando con mayor extensión de tierra y por tanto puede diversificar sus actividades primarias.

Sin embargo, los campesinos pueden insertarse en actividades fuera de la parcela agrícola para complementar sus ingresos. Por último, la agricultura familiar consolidada (AFC), en la que generalmente la producción es destinada solo a la venta y se caracteriza por tener mayor acceso a recursos agropecuarios que le permiten generar excedentes, y de esa forma, obtener capital que se destine a la producción.

Por su parte en México, se han clasificado dos tipos de AF (Yúnez et al., 2013; Schneider, 2014): agricultura familiar especializada (AFE), que refiere a las unidades de producción que obtienen más de $50 \%$ de su ingreso (bruto) total de actividades agrícolas y la agricultura familiar pluriactiva (AFP) integrada por unidades que obtienen $50 \%$ o menos de su ingreso en actividades agrícolas, mientras que el resto proviene de actividades no agrícolas.

El presente trabajo se realizó bajo un contexto de AFS y de carácter pluriactivo, por lo que se expondrán tres visiones dentro del área de la diversificación de los ingresos rurales. Algunos autores discuten que, dentro de los estilos de transformación estructural (TE), existen dos que llevan a los agentes productores del campo a diversificar sus actividades para la generación de ingresos (Rello y Saavedra, 2013).

El primer estilo de TE basa en una agricultura dual, cuyos rasgos son: emergencia de dos subsectores agrícolas, uno moderno vinculado al sector industrial y otro, con una producción rural pequeña encaminada al autoconsumo. Los ingresos agrícolas son bajos y los agentes se ven presionados a buscar otras fuentes de ingreso y empleo, el cual forma parte de un ejército industrial 
de reserva. El segundo estilo de TE se da en regiones pobres con escasos recursos: sólo hay un tipo de agricultura, la cual es muy débil y aporta sólo $20 \%$ del ingreso familiar, la estrategia de sobrevivencia es el empleo en zonas más dinámicas, así como el autoempleo y la migración.

Por esa razón sus actividades se encuentran ligadas principalmente a zonas de producción industrial, y prestadoras de servicios. De acuerdo con lo anterior, de estos dos estilos de TE, el primero se caracteriza por un desarrollo dual del campo y el segundo por una agricultura uniformemente pobre. En este tipo de agricultura emerge, en primer lugar, la necesidad de incorporarse a otros sectores de producción debido a lo magro de sus ingresos agrícolas, siendo el eje en común la diversificación de actividades como forma de sobrevivencia.

En segundo lugar, se sostiene que un incremento en las actividades no agrícolas no implica un mayor ingreso para los agentes económicos. Para mejorar el ingreso se debe incentivar la productividad agrícola, mediante variables como el capital, las tecnologías de producción, los precios de mercado, etc. La ausencia de estas variables ha generado un crecimiento desigual en el campo, y con ello los hogares con menores ingresos derivados de las actividades agrícolas son los que tienen más incentivos a diversificar (Escobal, 2001; Odoh y Nwibo, 2017).

Por último, hay hallazgos que evidencian que los productores rurales afrontan un proceso de desagrarización, debido a que el mayor peso de sus actividades se encuentra en ocupaciones fuera de la parcela agrícola (Mora y Cerón, 2015). Estas actividades se encuentran relacionadas con la migración a corto plazo debido a las nuevas características del mercado laboral (precarización del trabajo) donde se limita el establecimiento definitivo de los empleados en los lugares destino; se ocupan como jornaleros agrícolas o trabajadores de la construcción (Escalante et al., 2007).

En relación con el fenómeno de diversificación de ingresos o pluriactividad campesina, en este trabajo se define como la combinación de los diferentes empleos, oficios o negocios que se pueden crear como parte de las formas de sobrevivencia y esas decisiones dependen más de las condiciones del mercado de trabajo general que del mercado específico para los productos agrícolas (Martínez et al., 2018); es decir, los precios del maíz y semillas de calabaza en el caso de este trabajo. Cabe mencionar que en 2018 los campesinos de Tehuatzingo vendieron su cosecha a intermediarios que compraron en su comunidad el maíz a $\$ 3700.00 \mathrm{t}^{-1}$ y la semilla de calabaza a $\$ 40.00 \mathrm{~kg}^{-1}$.

Por otro lado, en la planta automotriz AUDI cercana a esta comunidad, el salario de un obrero para esas fechas osciló entre $\$ 250.00$ y $\$ 780.00$ por día, de manera que era más atractivo que la actividad agrícola por los ingresos inmediatos y más altos por jornada (e-consulta.com, 2017). La pluriactividad puede estar regida por el proceso de industrialización en el país. También cabe señalar que es un concepto muy relacionado con el proceso de desagrarización en el campo mexicano; es decir, a la disminución de la aportación de las actividades agrícolas en la generación de ingresos en el hogar (Jarquín et al., 2017).

Esta disminución hace según algunos estudios que en México la fuente de ingreso en los hogares rurales ya no provenga de actividades agropecuarias, sino de la participación en el trabajo asalariado, principalmente los servicios, la manufactura y la construcción (Cerón y Yúnez, 2015). Con base en lo anterior, el objetivo fue dimensionar las características sociodemográficas y la diversidad de los ingresos económicos de los hogares campesinos durante el año 2018, en Tehuatzingo, Libres, Puebla. 


\section{Materiales y métodos}

Para la selección del lugar de estudio se plantearon las siguientes premisas: en primer lugar, la existencia de una tradición agrícola arraigada en la comunidad como lo es el sistema milpa (SM), reflejo de los conocimientos ancestrales sobre la naturaleza, tecnología y prácticas agrícolas para garantizar la alimentación diversificada tradicional y sana (Sánchez y Romero, 2017). En segundo lugar, la producción de maíz como la actividad más importante.

En este sentido cabe resaltar al Distrito de Desarrollo Rural Libres entre los más competitivos en el estado de Puebla, es el que presenta mayor superficie sembrada y la única región que no tiene un déficit en el consumo de maíz en el estado (Flores et al., 2014).

El estudio se realizó en la comunidad de Tehuatzingo, que pertenece al municipio de Libres, Puebla. Este municipio se encuentra ubicado en la parte centro-norte del estado y comprende una superficie de $275 \mathrm{~km}^{2}$, lo que representa $0.8 \%$ de la superficie del estado. Se localiza entre los $19^{\circ}$ $27^{\prime} 52^{\prime \prime}$ de latitud norte y $97^{\circ} 41^{\prime} 15^{\prime}$ " de longitud oeste, con una altitud promedio de $2378 \mathrm{msnm}$, con precipitación entre 400 y $900 \mathrm{~mm}$ anuales (INEGI, 2017).

Tehuatzingo se fundó a partir del reparto agrario en el año 1929 con terrenos de cultivo que pertenecían a la hacienda de San Nicolás. Antes, todos vivían en el cerro llamado Rancho Viejo de donde son originarios. Actualmente son aproximadamente 507 habitantes (SEDESOL, 2010), de los cuales 100 son ejidatarios. La comunidad tiene alto grado de marginación y se ubica arriba de la media municipal que refleja la situación general del estado de Puebla con índice de marginación alto. Se ubica a $8 \mathrm{~km}$ de la ciudad de Libres, cabecera del municipio con el mismo nombre, que concentra 50\% de la población (a nivel municipal se registran 31520 habitantes) (INEGI, 2017).

A principios de 2018 se realizó la selección del lugar de estudio de acuerdo con lo citado anteriormente. Se contactó a las autoridades locales (comisariado ejidal y juez de paz) que fueron informantes clave y se llevó a cabo un recorrido de reconocimiento por la zona. Para la recolección de información primaria se tuvo como referencia la base de datos de ejidatarios que cuenta con 184 integrantes en total, de los cuales 100 pertenecen al ejido de Tehuatzingo y el 90\% cultiva maíz.

Para la evaluación de la diversificación de los ingresos rurales se utilizó la encuesta, por lo que se aplicó un cuestionario piloto para su valoración con cinco productores. Finalmente se realizaron adecuaciones a este instrumento y se aplicó durante el mes de julio de 2018 a una muestra de 46 ejidatarios de manera aleatoria simple. El tamaño de muestra se determinó con un nivel de confiabilidad de $95 \%$ y una precisión de $5 \%$ bajo la fórmula siguiente (Aguilar, 2005). $\mathrm{n}=\frac{\mathrm{N}-\mathrm{p} \cdot \mathrm{q}}{(\mathrm{N}-1) \mathrm{e}^{2}+\mathrm{Z}^{2} \mathrm{p} . \mathrm{q}}$; donde: $\mathrm{N}=$ tamaño de la población (90); $\mathrm{Z}=$ nivel de confianza $(95 \%) ; \mathrm{p}=$ probabilidad de que la muestra sea representativa; $\mathrm{q}=$ probabilidad de que la muestra no sea representativa; e= error máximo (10\%); $\mathrm{n}=$ tamaño de muestra (46).

Para medir la diversificación de ingresos se utilizó el índice de Simpson (Ds) el cual fue desarrollado por Simpson en 1949 con la finalidad de medir la diversidad y abundancia de las especies (Mora y Cerón, 2015). En este trabajo se calculó dicho índice que muestra los resultados diferenciados según el tipo de manejo: monocultivo y policultivo. El Ds se mide en un rango de 0 y 1 , indica que entre más se acerca a 1 existe una mayor fuente de ingresos en los hogares rurales. 
Además, prevalece una mayor diversificación en el ingreso generado por cada una de estas actividades, mientras si se acerca a 0 , el ingreso es generado a partir de una sola actividad y se calcula como: $\mathrm{D}_{\mathrm{s}}=1-\sum \mathrm{p}_{\mathrm{i}}^{2}$; donde: $\mathrm{P}_{\mathrm{i}}=$ proporción de la categoría $\mathrm{i}$, (en este caso, el ingreso procedente de la fuente i) en el ingreso total del hogar: $p_{i=y_{i}} \sum_{i=I}^{I} y_{i}$.

Las variables utilizadas para hacer la medición del Ds fueron los ingresos obtenidos por la producción de maíz, calabaza y frijol, dependiendo de la existencia o no en cada una de las parcelas. Para obtener los ingresos se multiplicó el rendimiento total de cada cultivo por el precio actual de kilogramo en el momento de aplicar la encuesta. Otra variable utilizada fue ingreso por pacas de rastrojo, el ingreso derivado de maíz (tortillas y hojas para tamal), los ingresos por actividades fuera de la parcela (divididas en primarias, secundarias y terciarias) y otras fuentes de ingreso en el hogar derivado del trabajo de algún familiar ya sea dentro de la comunidad o en el exterior.

Además, se cuantificó el impacto de los sistemas de manejo del agroecosistema maíz en la diversificación del ingreso mediante un modelo logit, ordenado donde la variable dependiente es categórica. Nuestra variable dependiente observada (y) es el monto reportado de ingreso no agrícola que se ha convertido a un puntaje z y se han definido las categorías: baja, media baja, media alta y alta, tomando como valores límite el primer cuartil, la mediana y el tercer cuartil como se muestra en el Cuadro 1.

Cuadro 1. Clasificación de niveles de diversificación, acorde al z-score del monto de ingresos no agrícolas reportados.

\begin{tabular}{cc}
\hline Nivel de diversificación & Características \\
\hline$[y=1]$ baja & Si z-score $\leq$ percentil 25 \\
{$[y=2]$ media baja } & Si percentil $25<$ z-score $\leq$ percentil 50 \\
{$[y=3]$ media alta } & Si percentil $50<$ z-score $\leq$ percentil 75 \\
{$[y=4]$ alta } & Si z-score $>$ percentil 75 \\
\hline
\end{tabular}

La categoría 1 (baja) representa un bajo nivel de generación de ingresos no agrícolas (un productor que reporte este valor presenta alta especialización en la generación de ingresos agrícolas y muy baja capacidad de diversificación de sus actividades); mientras que la categoría 4 (alta) representa el mayor nivel de generación de ingresos no agrícolas (un productor que reporte este valor presenta una menor especialización en la generación de ingresos agrícolas y mayor capacidad de diversificación de sus ingresos hacia otras fuentes no agrícolas). Por lo anterior, esta variable categórica observada (y) mide los niveles de diversificación de ingresos hacia fuentes no agrícolas.

El objetivo del modelo es estimar cómo cambian las probabilidades de reportar mayor diversificación hacia fuentes no agrícolas según el sistema de manejo controlando los efectos de las variables edad, educación, superficie y sistema de manejo, siendo estas las variables explicativas. La variable dependiente implica los ingresos no agrícolas.

El modelo logit ordenado (Long y Freese, 2001) asume que la probabilidad de observar cierto valor de $\mathrm{y}(\operatorname{Pr}[\mathrm{y}=\mathrm{m}])$ es condicional a un conjunto de regresores y se define por la expresión: $\operatorname{Pr}$ $[\mathrm{y}=\mathrm{m} / \mathrm{x}]=\operatorname{Pr}\left[\tau_{\mathrm{m}-1} \leq \mathrm{y}^{*}<\tau_{\mathrm{m}} \mid \mathrm{x}\right]$; donde: $\mathrm{y}^{*}=$ es una variable subyacente que toma valores entre (- 
$\infty,+\infty$ ) y condiciona los posibles valores observados y se vinculan a través de la siguiente regla: $\mathrm{y}=1$ si $^{*}<\tau_{1 ;} \mathrm{y}=2 \quad$ sí $\tau \leq y *<\tau_{12} ; \mathrm{y}=3$ sí $\tau_{2} \leq y *<\tau_{3} ; \mathrm{y}=4$ si $\mathrm{y}^{*} \geq \tau_{3} ;$ Los valores de $\mathrm{y}^{*}$ son determinados por la función lineal: $y *=\hat{\beta}_{1}+\hat{\beta}_{2}$ Superficie $+\hat{\beta}_{3} \operatorname{Edad}+\hat{\beta}_{4}$ Educación $+\hat{\beta}_{5}$ Sistema + e.

Estos valores incluyen los siguientes factores determinantes captados por la encuesta de manera que se validan para saber si influyen en el comportamiento de los niveles de diversificación de ingresos. Las variables explicativas del modelo son: edad del entrevistado, medida en años cumplidos. Educación, representada por los años de escolaridad concluidos de cada uno de los productores. Superficie, extensión de tierra cultivable (medida en hectáreas). Sistema de manejo, variable binaria que señala $0=$ si el productor reportó monocultivo; $1=$ si el productor reportó policultivo (SMM) y por último ingreso agrícola, que indica la proporción de los ingresos totales generados por las actividades agrícolas.

\section{Resultados y discusión}

\section{Sistema de producción de maíz en monocultivo (SMo)}

En este grupo la muestra representativa fue de 16 productores $(35 \%)$ del total de entrevistados con una edad promedio de 51 años y 6 años de escolaridad. La siembra de maíz en monocultivo implica sembrar una sola especie. Si bien siguen conservando variedades criollas, ocasionalmente en este sistema de cultivo se hace uso de herbicidas para el control de arvenses, aunque esta práctica sólo la realizaron $18 \%$ de los productores entrevistados, mientras que el resto controla las hierbas a través del uso de técnicas tradicionales como las labores de cultivo (escarda, labra, etc.).

\section{Sistema milpa modificado (SMM)}

Se le llama sistema milpa modificado debido a que en este tipo de manejo solo combinan dos cultivos: maíz y calabaza; a diferencia del sistema milpa tradicional en el que se intercala maíz con alguna leguminosa como frijol, haba o ayocote y calabaza. En este grupo se encuentra $65 \%$ de campesinos del total de la muestra; la edad promedio de los agricultores es de 55 años, mientras que en promedio tienen 5 años de escolaridad (Cuadro 2). Estos resultados son similares a lo que presentan Ramos et al. (2013) para la región de Libres, donde la edad que predomina es por arriba de los 55 años y la escolaridad promedio fueron 4 años de estudio.

Cuadro 2. Características generales por sistema de manejo.

\begin{tabular}{ccc}
\hline & Monocultivo & Milpa modificada \\
\hline Edad (años) & 51 & 55 \\
Escolaridad (años) & 6 & 5 \\
& Sexo (porcentaje) & \\
Femenino & 12 & 13 \\
Masculino & 88 & 87 \\
Número de hijos & 4 & 5 \\
Superficie promedio (ha) & 1.5 & 2 \\
\hline
\end{tabular}




\begin{tabular}{ccc}
\hline & Monocultivo & Milpa modificada \\
\hline Maíz & Producción (rendimiento kg ha $\left.{ }^{-1}\right)$ & 2185 \\
Calabaza & 2656 & 201 \\
& 0 & \\
Comerciante & Otras actividades (porcentaje) & 7 \\
Construcción & 25 & 20 \\
Ganadería & 13 & 13 \\
Jornalero & 6 & 20 \\
Obrero & 6 & 7 \\
Ninguna & 6 & 33 \\
Total & 44 & 100 \\
\hline
\end{tabular}

Elaborado con base en la información de campo 2018.

En el grupo de SMo, la actividad complementaria de mayor importancia fue el comercio por cuenta propia, desde pequeños abastecimientos de abarrotes hasta el servicio de venta de comida mientras que en el SMM las labores complementarias son la construcción que en su mayoría se realiza fuera de la comunidad por periodos temporales, y hasta otros estados del país, así como el trabajo de jornaleros, que a diferencia de flujos migratorios pasados se les permitía establecerse en las ciudades destino, en la actualidad es complicado por la precariedad de los empleos disponibles.

Conforme a la categorización el SMM presenta 57\% del total de entrevistados con grado de diversificación baja (categoría 2), seguido de $20 \%$ contemplado con una alta diversificación (categoría 4). A diferencia, en el SMo la mayoría de los productores se ubica en la categoría 3 lo cual significa que la diversificación de sus actividades es media, pero con tendencia a la diversificación baja y muy baja (Cuadro 3).

Cuadro 3. Proporción de diversificación por sistema de manejo.

\begin{tabular}{cccc}
\hline \multirow{2}{*}{ Categoría } & \multicolumn{2}{c}{ Sistema de manejo } & \multirow{2}{*}{ Total $(\mathrm{n}=46)$} \\
\cline { 2 - 3 } & $\mathrm{SMM}(\mathrm{n}=30)$ & $\mathrm{SMo}(\mathrm{n}=16)$ & 15 \\
1 & 10 & 25 & 46 \\
2 & 57 & 25 & 22 \\
3 & 13 & 37.5 & 17 \\
4 & 20 & 12.5 & 100 \\
Suma & 100 & 100 & \\
\hline
\end{tabular}

Elaborado con base en la información de campo.

Las características básicas de las variables utilizadas en el modelo y las estadísticas descriptivas para el tamaño de muestra de 46 productores se presentan en el Cuadro 4. 
Cuadro 4. Resumen de las variables a trabajar en el modelo.

\begin{tabular}{cccccc}
\hline Variable & $\mathrm{n}$ & Media & Desviación estándar & Min & Max \\
\hline Edad & 46 & 54 & 14.1 & 25 & 84 \\
Educación & 46 & 5.6 & 3.9 & 0 & 16 \\
Superficie (ha) & 46 & 1.8 & 1 & 0.5 & 5 \\
Sistema de manejo & 46 & 0.4 & 0.5 & 0 & 1 \\
Porcentaje de ingreso agrícola & 46 & 51.3 & 33.4 & 2.2 & 100 \\
\hline
\end{tabular}

Elaborado con base en la información de campo 2018.

El ingreso no agrícola (variable dependiente), tiene una media de 43675.6 lo que significa que los ingresos promedios fuera de la parcela agrícola con una desviación estándar de 42736.7 y una varianza de 1.8. En la Figura 1 se observa que existe poca diferencia entre los ingresos no agrícolas de los dos grupos de manejo. La media para el SMo es de $49095 \pm 50359.5$ y para el SMM el promedio de las ganancias fuera de la parcela agrícola es de $43675.9 \pm 38$ 697.9.

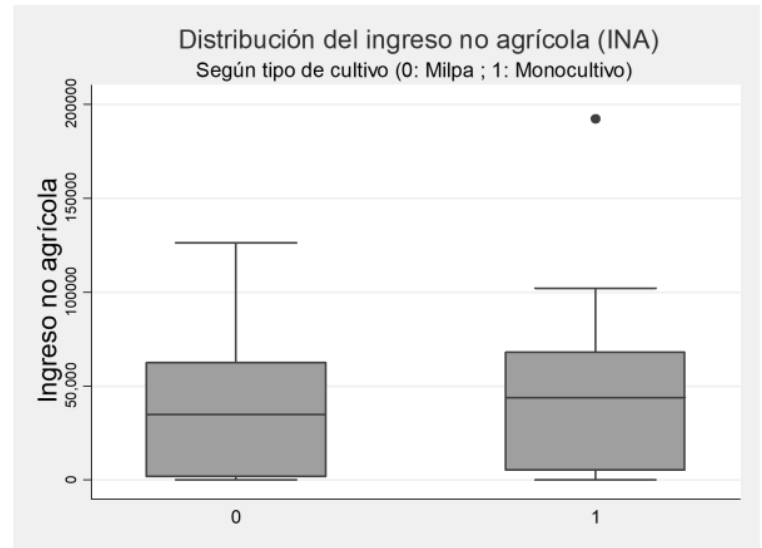

Figura 1. Distribución del ingreso no agrícola en un ciclo de cultivo.

La distribución de los menores ingresos no agrícolas se da entre el percentil del 26-50\%. Mientras que los mayores ingresos no agrícolas están ubicados en el percentil del 76-100\%. Existe también un dato atípico el cual se encuentra en el grupo de policultivo (SMM). Mediante un modelo logit ordenado se obtuvo el valor de $\mathrm{chi}^{2}=0$ el cual indica que los coeficientes son conjuntamente significativos. Mientras que la Pseudo $\mathrm{R}^{2}$, cercana a $\mathrm{r}^{2}$ tradicional indica que $27 \%$ de la variable dependiente (diversificación del ingreso rural) puede ser explicada por las variaciones de las variables independientes (Cuadro 5).

Cuadro 5. Resultados básicos del modelo: regresión logística ordenada.

\begin{tabular}{ccccc}
\hline Variable & Coeficiente & $\mathrm{z}$ & $\mathrm{P}>[\mathrm{z}]$ & Error estándar \\
\hline Edad & $-0.0543^{* *}$ & -2.08 & 0.038 & 0.0261 \\
Educación & -0.0806 & -0.57 & 0.572 & 0.1426 \\
Superficie & $2.3213^{* *}$ & 3.88 & 0 & 0.5977 \\
Sistema de manejo & -0.7715 & -1.22 & 0.222 & 0.6319 \\
Porcentaje de ingreso agrícola & $-0.0721^{* *}$ & -3.9 & 0 & 0.0185 \\
\hline
\end{tabular}

Elaborado con base en la información de campo 2018. $\mathrm{N}=46$; Pseudo $\mathrm{R}^{2}=0.2755 ;{ }^{* *}=\operatorname{significativa}$ al $1 \%$. 
El nivel de escolaridad no explica que los productores diversifiquen o no debido a su significancia estadística mayor a 0.05 , este resultado puede variar según el tamaño de muestra. Además, contrasta con literatura que considera la educación como un aspecto favorable para el impulso a los hogares en la realización de actividades no agropecuarias (De Janvry y Sadoulet, 2004; Yúnez y Meléndez, 2007).

Por otra parte, De Grammont (2009a) encontró que el nivel de educación no es suficiente para lograr un aumento en los ingresos de los productores, pues se han hallado casos en los niveles más bajos de ingresos en que los agricultores cuentan con altos niveles de educación; el nivel educativo no es un factor determinante para aumentar los ingresos económicos del productor. De igual forma no hay diferencia significativa entre los sistemas de manejo, puesto que existe muy poca variación entre los ingresos de cada uno de los grupos.

Por el contrario, las variables edad y tamaño de la superficie son factores determinantes para que los productores elijan diversificar sus actividades. Con relación a la variable edad hay una relación negativa en cuanto a la diversificación de los ingresos no agrícolas; es decir, a mayor edad existe menor probabilidad de que tiendan a realizar otras actividades, lo cual se asemeja al trabajo realizado por Andersen y Valencia (2010) donde concluyen que las personas mayores de edad tienden a limitarse al trabajo agrícola.

El tamaño de la superficie cultivada influye en las probabilidades de diversificación de las actividades rurales, es así como a menor extensión de cultivos existe una mayor probabilidad de participar en actividades no agrícolas (Andersen y Valencia, 2010). Por otra parte, se encontró que a mayor proporción de ingresos agrícolas menos incentivos tiene a reportar otras actividades de índole no agrícola, esto se relaciona con lo mencionado por De Grammont (2009b) donde refiere que la dinámica de la pluriactividad cambia según el nivel de ingreso.

En otros términos, a menores incentivos económicos mayores son las prácticas en los hogares rurales para complementar su ingreso. Incluso, menciona que esta tendencia de diversificación de actividades en el campo mexicano, se acompaña de la caída de los precios del maíz, así como de los salarios. Entre las variables edad y educación se detectó correlación negativa y significativa con un nivel de significancia de 5\% $(\mathrm{rho}=-0.8198)$, dicha asociación lineal entre variables explicativas genera un problema de alta colinealidad en el modelo econométrico. Con fines de evitar el sesgo en la estimación de las varianzas de los coeficientes beta del modelo logit, es necesario eliminar dicha correlación entre regresores.

Por esa razón se atendió el problema mediante una depuración de la correlación con una regresión auxiliar, donde obtenemos el componente puro de variaciones de la edad que no resultaba linealmente dependiente de la educación, una vez extraído dicho componente la correlación entre ambas variables ha sido eliminada (Gujarati y Porter, 2010).

Se realizó un análisis de forma ilustrativa para ahondar en las correlaciones que son significativas (Figura 2), en el primer grupo correspondiente al monocultivo, la diversificación alta de las actividades no agrícolas tiende a disminuir conforme la edad aumenta, también se puede observar que en este grupo el tipo de diversificación que domina es la de tipo medio-bajo entre los 40-60 años y entre más se acerca a los 20 años las actividades agrícolas realizadas son más diversas. 


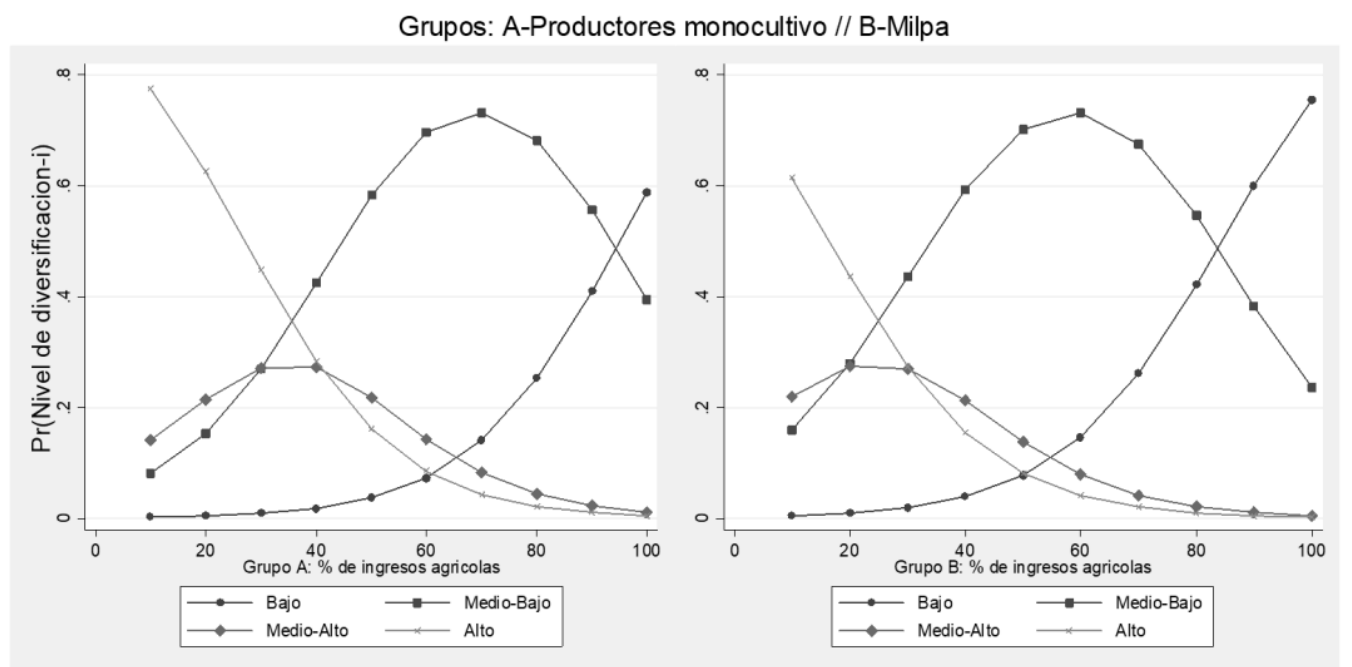

Grupos A, B: Edad 53 años, escolaridad: 6 años, Superficie promedio 1.8 ha

Figura 2. Probabilidades estimadas de reportar cada nivel de diversificación según edad.

En cuanto al grupo que corresponde al sistema de milpa modificada, la diversificación de las actividades no agrícolas también se concentra en un nivel medio-bajo, lo cual reafirma que las probabilidades a reportar para una diversificación alta en actividades fuera del predio agrícola son escasas y casi nulas cuando se acerca a una edad de ochenta años en ambos grupos de productores. En la Figura 3 se observan los resultados obtenidos a través del modelo de las probabilidades de los niveles de diversificación de los ingresos no agrícolas, considerando que la variable que ahora cambia es el porcentaje de ingresos provenientes de actividades agrícolas.

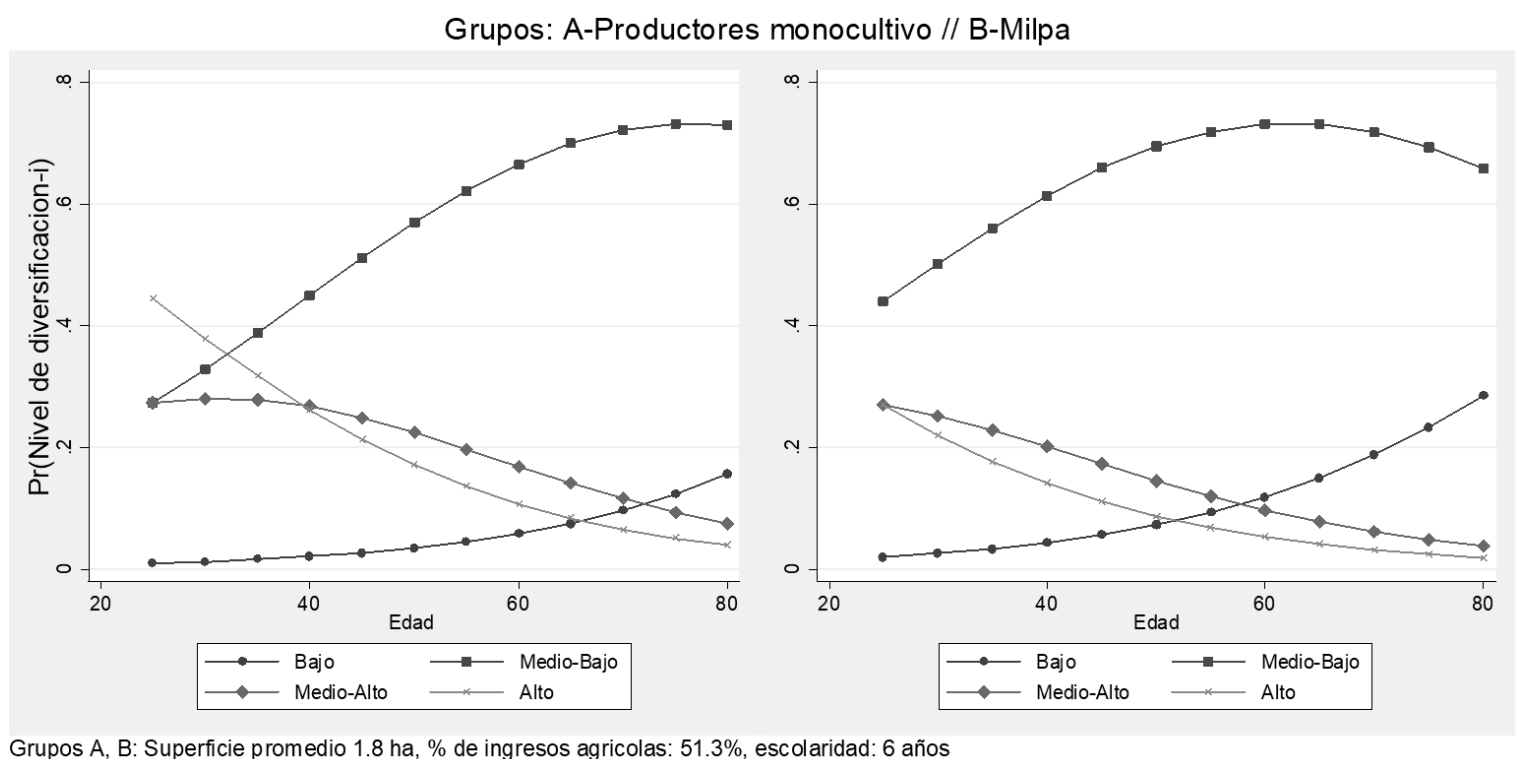

Figura 3. Probabilidades de reportar cada nivel de diversificación según porcentaje de ingresos agrícolas. 
En el grupo de monocultivo al igual que en la milpa modificada, el nivel de diversificación disminuye cuando el porcentaje de ingresos derivado de actividades agrícolas aumenta, es decir que a mayor nivel de ingresos generados en la parcela agrícola los productores tienden a no buscar otras fuentes de ingreso, lo cual coincide con De Grammont (2009c) donde menciona que la pluriactividad difiere según el nivel de ingresos, por lo que en los niveles más altos, la diversificación de actividades desaparece. También se comprueba que el nivel de diversificación en actividades fuera del predio agrícola es mayor en el sistema de producción en monocultivo.

\section{Índice de Simpson}

El Índice de Simpson para el sistema milpa modificado reporta mayor número de actividades que aportan al ingreso de la unidad campesina en comparación con el sistema en monocultivo, debido a que el Ds es cercano a 1 (Cuadro 6).

Cuadro 6. Comparación del índice de Simpson entre los dos sistemas de manejo.

\begin{tabular}{cc}
\hline Sistema & Índice de Simpson \\
\hline Monocultivo & 0.912 \\
Milpa modificado & 0.958 \\
\hline
\end{tabular}

Elaborado con base en la información de campo 2018.

El ingreso agrícola es mayor en el SMM dado que representa 54\% de sus ingresos totales, lo cual concuerda con el Ds debido a que realizan actividades que se encuentran mayormente ligadas a la parcela ejidal para asegurar el sustento, y al mismo tiempo diversifican sus cultivos combinando maíz con calabaza. Sucede lo contario en el SMo debido a que la proporción de ingresos que no depende de actividades agrícolas es mayor en este tipo de manejo, debido a que asciende a 56\% mientras en el SMM los ingresos no agrícolas representan $46 \%$.

Del total de la muestra de productores $(n=46), 29$ se dedican a otras actividades además de la agricultura, lo que en términos porcentuales representa 63\%. Si se desglosan los resultados por sistema de manejo, en el sistema en monocultivo $56 \%$ de los productores realiza otra actividad, y en el sistema milpa modificado $67 \%$ se dedica a otras actividades además del trabajo en el predio agrícola. Acorde a Magdaleno et al. (2014) esto refleja el ajuste a las prácticas que las familias campesinas realizan para continuar con sus formas de subsistencia, a consecuencia de la falta de atención en el agro mexicano.

De acuerdo con lo anterior, en el Cuadro 7 se muestran las principales fuentes de ingresos fuera de la parcela de cultivo en el SMo, los cuales provienen del empleo por cuenta propia, seguido por el empleo asalariado, ambos derivados de actividades no agrícolas. Mientras que en el SMM existe un ingreso proveniente del empleo asalariado de la rama agrícola como los que realizan trabajos con la yunta y jornaleros. Cabe destacar que en este grupo de productores domina el empleo no agrícola asalariado, donde principalmente se ubica el ramo de la construcción o la industria obrera, siendo la principal empresa en la región la automotriz de carros de lujo AUDI. 
Cuadro 7. Diversificación del empleo en Tehuatzingo.

\begin{tabular}{|c|c|c|c|c|}
\hline \multirow{2}{*}{$\begin{array}{l}\text { Sistema de } \\
\text { producción }\end{array}$} & \multicolumn{2}{|c|}{ Empleo por cuenta propia $(\%)$} & \multicolumn{2}{|c|}{ Empleo asalariado (\%) } \\
\hline & $\begin{array}{l}\text { Actividades } \\
\text { agrícolas }\end{array}$ & $\begin{array}{l}\text { Actividades no } \\
\text { agrícolas }\end{array}$ & $\begin{array}{c}\text { Actividades } \\
\text { agrícolas }\end{array}$ & $\begin{array}{l}\text { Actividades no } \\
\text { agrícolas }\end{array}$ \\
\hline SMo & 11 & 45 & 11 & 33 \\
\hline SMM & 20 & 10 & 30 & 40 \\
\hline
\end{tabular}

Elaborado con base en la información de campo 2018.

Por lo menos 55\% del total del ingreso en el hogar campesino proviene de actividades no agrícolas que son el apoyo para mantener la familia y poder hacer su siembra, esto coincide con lo encontrado por Janvry y Sadoulet en un estudio de 2004 donde mencionan que el ingreso generado por actividades realizadas fuera del predio agrícola representa más de la mitad de los ingresos de los hogares campesinos en México.

La pluriactividad es una práctica campesina que emplean para continuar sembrando, utilizan su mano de obra en empleo temporal, jornaleros agrícolas, en la construcción y el empleo por cuenta propia como el comercio. Esto coincide con lo que describen Magdaleno et al. (2014) así como Escalante et al. (2007) donde las familias complementan su ingreso agrícola con actividades no agrícolas. Es así que existe un declive en las actividades que tradicionalmente se realizaban en el campo mexicano, donde factores como la manufactura y el sector servicios impactan en la reducción de la producción agrícola.

\section{Conclusiones}

Las familias campesinas utilizan diferentes prácticas para mantener la siembra de maíz con la finalidad de obtener el sustento para la familia. Los campesinos que diversifican sus cultivos tienden a generar mayores ingresos agrícolas en comparación a los que se dedican exclusivamente a solo un cultivo. Más de la mitad de los productores de ambos grupos, desarrollan otras actividades aparte de la agricultura como mecanismos para asegurar la permanencia y reproducción de la familia.

El SMM presenta mayor índice de diversificación de ingresos agrícolas que el SMo de acuerdo al índice de Simpson. Asimismo, la edad más propensa para diversificar sus ingresos es entre 20 y 30 años, mientras que productores con mayor edad diversifican menos. Con respecto a los ingresos no agrícolas, la principal fuente proviene de la participación en el mercado de trabajo asalariado, lo cual también es una tendencia actual en el campo mexicano. En Tehuatzingo los campesinos se emplean temporalmente como jornaleros o en la industria de la construcción. Actualmente las condiciones laborales son precarias e inseguras, incapaces de garantizar estabilidad económica a la mano de obra sobrante del campo.

\section{Literatura citada}

Aguilar, S. 2005. Fórmulas para el cálculo de la muestra en investigaciones de salud. México. Salud de Tabasco. 2(11):333-338. 
Alves, P. and Días, P. 2019. Family farming in agroecological transition: a look at the marketing of milk and dairy products in municipalities of the Zona da Mata of Minas Gerais State, Brazil. Brasil. Ciencia Rural. 7(49):1-8. http://dx.doi.org/10.1590/0103-8478cr20180299.

Andersen, L. E. y Valencia, H. 2010. Trabajo no agrícola de las familias rurales de Bolivia: un análisis de determinantes y efectos. Serie de documentos de Trabajo sobre desarrollo No. 01-2010. IDRC-Universidad Católica Boliviana. 19 p.

Bosc, P. M. and Sourisseau, J. M. 2019. Sustainable rural livelihoods to analyze family farming dynamics: a comparative perspective. Japón. The Natural Resource Economics Review. 4(1):35-49

Cerón, H. y Yúnez, A; Rivera, F.; Chávez, A.; Mora, J. J. y Taylor, J. E. 2015. Diversificación en la economía rural: hacia actividades no agropecuarias y sus impactos en pobreza y desigualdad. In: la economía del campo mexicano: tendencias y retos para su desarrollo. El Colegio de México. México, DF. 117-156 pp.

De-Grammont, H. 2009a. Boom agrícola y persistencia de la pobreza rural en México. In: Boom agrícola y persistencia de la pobreza rural: estudio de ocho casos. Da-Silva, J. G; Gómez, S. y Castañeda, R. (Ed.). FAO. Roma, Italia. 225-263 pp.

De-Grammont, H. 2009b. La desagrarización del campo mexicano. México. Convergencia. Revista de Ciencias Sociales. 50(16):13-55.

De-Grammont, H. 2009c. La nueva estructura ocupacional en los hogares rurales mexicanos. In: la pluriactividad en el campo latinoamericano. De Grammont, H. y Martínez, L. (Coords.). FLACSO. Quito, Ecuador. 273-307 pp.

De-Janvry. y Sadoulet. 2004. Estrategias de ingresos de los hogares rurales de México: el papel de las actividades desarrolladas fuera del predio. In: empleo e ingresos rurales no agrícolas en América Latina. CEPAL-FAO-RIMISP. Santiago, Chile. 107-148 pp.

E-consulta.com. 2017. Obreros de Audi ganarán hasta 780 pesos diarios durante 2018. https://www.e-consulta.com/nota/2017-12-22/economia/obreros-de-audi-ganaran-hasta780-pesos-diarios-durante-2018.

Escalante, R.; Catalán, H.; Galindo, L. M. y Reyes, O. 2007. Desagrarización en México: tendencias actuales y retos hacia el futuro. Colombia. Cuadernos de Desarrollo Rural. 59(4):87-116.

Escobal, J. 2001. The determinants of nonfarm income diversification in rural Peru. Gran Bretaña. World Development. 3(29):497-508. https://doi.org/10.1016/S0305-750X(00)00104-2.

Flores, L. A.; García, J. A.; Mora, J. S. y Pérez, F. 2014. Producción de maíz (Zea mays L.) en el estado de Puebla: un enfoque de equilibrio espacial para identificar las zonas más competitivas. México. Agric. Soc. Des. 2(11):223-239.

García, J. C.; Gutiérrez, J. G.; Balderas, M. A. y Araújo, M. R. 2016. Estrategia de vida en el medio rural del altiplano central mexicano: el huerto familiar. México. Agric. Soc. Des. 4(13):621-641.

Gujarati, D. N. y Porter, D. C. 2010. Econometría. Mc Graw Hill. Quinta edición. México, DF. 320-351 pp.

INEGI. 2017. Instituto Nacional de Estadística, Geografía e Informática. Anuario estadístico y geográfico de Puebla. https://www.datatur.sectur.gob.mx/itxef_docs/pue_anuario_pdf.pdf.

Jarquín, N. H.; Castellanos, J. A. y Sangerman-Jarquín, D. M. 2017. Pluriactividad y agricultura familiar: retos del desarrollo rural en México. México. Rev. Mex. Cienc. Agríc. 4(8):949-963. 
Long, J. S. and Freese, J. 2001. Regression models for categorical dependent variables using STATA. Stata Press Publication. College Station, Texas. 337 p.

Magdaleno, E.; Jiménez, M. A.; Martínez, T. y Cruz, B. 2014. Estrategias de las familias campesinas en Pueblo Nuevo, Municipio de Acambay, Estado de México. México. Agric. Soc. Des. 2(11):167-179.

Martínez, M.; De-Souza, M. y Mora, J. 2018. Cambios en el empleo e ingreso de los hogares rurales de México, 2002-2007. México. Región y Sociedad. 71(30):1-29. https://doi.org/10.22198/rys.2018.71.a772.

Mora, J. J. y Cerón, H. 2015. Diversificación de ingresos en el sector rural y su impacto en la eficiencia: evidencia para México. Cuadernos de Desarrollo Rural. 76(12):57-81.

Odoh, N. E. and Nwibo, S. U. 2017. Socio-economic determinants of rural non-farm households income diversification in Southeast Nigeria. Inter. Res. J. Finance Econ. 164(1):116-128.

Orsini, G.; Domínguez, N. y Serfaty, N. 2018. Asociativismo y agro: el caso de las familias productoras en Entre Ríos, Argentina. SaberEs. 1(10):67-85.

Ramos, J. G.; Jaramillo, J. L.; Parra, F. y González, G. J. 2013. Factores que determinan la persistencia de la producción campesina de maíz: el caso del municipio de Libres, Puebla. México. Ra Ximhai Revista de Sociedad, Cultura y Desarrollo Sustentable. 1(9):15-28. 10.35197/rx.09.01.e.2013.02.jr.

Rello, F. y Saavedra, F. 2013. Diversificación productiva y transformación estructural en México: estudios de caso de tres regiones. Investigación Económica. 284(72):111-129. http://dx.doi.org/10.1016/S0185-1667(13)72594-3.

Sabourin, E.; Samper, M.; Le-Coq, J. F.; Massardier, G.; Sotomayor, O. y Marzin, J. 2015. Análisis transversal de las políticas sobre agricultura familiar en América Latina. In: políticas públicas y agriculturas familiares en América Latina y el Caribe: balance, desafíos y perspectivas. Sabourin, E.; Samper, M. y Sotomayor, O. (coords.). CEPAL. San José, Costa Rica. 19-44 pp.

Sánchez, M. P. y Romero, A. O. 2017. El sistema milpa y la producción de maíz en la agricultura campesina e indígena de Tlaxcala. Benemérita Universidad Autónoma de Puebla. Primera edición. México. 241 p.

Schejtman, A. 2008. Alcances sobre la agricultura familiar en América Latina. In: documento de trabajo No. 21. Programa Dinámicas Territoriales Rurales. RIMISP. Santiago, Chile. 41 p.

Schneider, S. 2003. A pluriatividade na agricultura familiar. Editora da UFRGS. $2^{\text {nd }}$. (Ed). Porto Alegre, Brasil. 255 p.

Schneider, S. 2014. La agricultura familiar en América Latina: un nuevo análisis comparativo. Fondo Internacional de Desarrollo Agrícola (FIDA). Roma, Italia. 4-32 pp.

SEDESOL. 2010. Secretaría de Desarrollo Social. Catálogo de localidades. http://www.microrregiones.gob.mx/catloc/LocdeMun.aspx ?buscar=1\&tipo=nombre\&cam po=loc \&valor $=$ tehuatzingo.

Woods, M. 2014. Family farming in the global countryside. Reino Unido. Anthropological notebooks, 3(20):31-48.

Yúnez, A. y Meléndez, A. 2007. Efectos de los activos familiares en la selección de actividades y en el ingreso de los hogares rurales de México. México. Investigación económica. 260(66):49-80.

Yúnez, A.; Cisneros, A. I. y Meza, P. 2013. Situando la agricultura familiar en México: Principales características y tipología. RIMISP. Serie documentos de trabajo $\mathrm{N}^{\circ} 149$. Santiago, Chile. $40 \mathrm{p}$. 